\title{
ENTROPY GENERATION RATE IN UNSTEADY BUOYANCY-DRIVEN HYDROMAGNETIC COUPLE STRESS FLUID FLOW THROUGH A POROUS CHANNEL
}

\author{
S.O. Kareem ${ }^{1}$, S.O. Adesanya ${ }^{2}$, J.A. Falade ${ }^{3}$, U.E. Vincent ${ }^{4}$ \\ ${ }^{1,3,4}$ Department of Physical Sciences \\ Redeemer's University \\ NIGERIA \\ ${ }^{2}$ Department of Mathematical Sciences \\ Redeemer's University \\ NIGERIA \\ ${ }^{2}$ Department of Mathematics \\ Vaal University of Technology \\ Vanderbijlpark 1911 \\ SOUTH AFRICA \\ ${ }^{4}$ Department of Physics \\ Lancaster University \\ Lancaster LA1 4YB, UNITED KINGDOM
}

\begin{abstract}
In this paper, the entropy generation rate in unsteady buoyancy-driven hydromagnetic couple stress fluid flow through a porous channel has been investigated. The partial differential equations are converted into their corresponding dimensionless equivalence, including the prescribed initial boundary conditions. These equations were solved using the Adomian decomposition method and the behaviour of some pertinent fluid variables, such as velocity, temperature, entropy generation rate and the irreversibility ratio were examined and discussed for different parameters of interest, which include, the Grashof number, the Hartmann's number, the Reynolds number, the Brinkman number and the couple stress pa-
\end{abstract}

\footnotetext{
Received: January 16, 2017

Revised: $\quad$ May 21, 2017

Published: July 14, 2017

$\S_{\text {Correspondence author }}$
}

(c) 2017 Academic Publications, Ltd. url: www.acadpubl.eu 
rameter. It was found that the entropy generation rate in the fluid model largely depends on the intermolecular forces between the fluid parcels. This has accounted for the observed increase in the entropy generation rate with respect to the couple stress parameter. Other observed entropy generation rate trends found their cause to the internal phenomenon in the fluid, though may be triggered by other forces such as the buoyancy and the Lorentz forces. Graphs are shown to illustrate the findings.

AMS Subject Classification: 76D05, 35Q30

Key Words: magnetic field, entropy generation, unsteady flow, irreversibility ratio, buoyancy flow

\section{Introduction}

The study of hydromagnetic flow of fluid that exhibits a non-symmetric stress tensor in its fluid structure [1] has been on the increase due to its numerous applications in engineering and industries. For instance, in geophysical prospecting including geothermal and petroleum reservoirs, and molten metal processing. This class of fluid, which is generally referred to as non-Newtonian fluid, includes lubricating films with polymer additives, molten metals and animal blood $[2,3]$. Another example of non-Newtonian fluid is the fluid carrying charged particles, which are subjected to electomagnetic field that causes the particles to rotate relative to thier neighbours [4].

In this present studies, emphasis is laid on the flow of non-Newtonian couple stress fluid, whose convective motion is influenced by a uniform transverse magnectic field. In this direction, appreciable number of studies have been reported in the literature over the years. For instance, Shercliff [5] investigated the steady motion of an electrically conducting viscous fluid along channels in the presence of an imposed transverse magnetic field when the walls do not conduct currents. Adesanya and Makinde [6] studied the effect of radiative heat transfer to oscillatory hydromagnetic non-Newtonian couple stress fluid flow through a porous channel with non-uniform wall temperature due to periodic heat input at the heated wall. In a related work, Reza and Gupta [7] examined the flow of a combination of incompressible viscous electrically conducting fluids in the presence of a transverse uniform magnetic field. Das and Jana [8] presented the second law analysis for magnetohydrodynamic incompressible fluid flow through a porous channel by imposing Navier slip conditions at the walls. Adesanya and Falade [9] analysed the inherent irreversibility in the flow of hydrodynamic third grade fluid through a channel saturated with porous materials. Other important studies in this direction include $[10,11,12,13,14,15,16,17,18,19]$ 
However, none of the references mentioned above on the analysis and entropy generation of non-Newtonian couple stress magnetohydromagnetic fluid flow has considered the analysis of entropy generation rate in magnetohydrodynamic couple stress fluid flow in porous channel. In reality, non-Newtonian couple stress fluid are used as working fluids in many technological and industrial processes, including polymer technology, petroleum industry [20]. Motivated by studies in $[6,7,8,9,21,10,11,12,13,14,15,16,17,18,19]$, the objective of the present study is to examine the influence of magnetic field and Ohmic heating of the couple stress fluid on the entropy production within the flow channel, which has not been accounted for in the literature. The outcoming results is expected to enhance many industrial and thermal engineering processes whose working medium is non-Newtonian fluid, with a view to minimise entropy generation which tends to deplete the amount of available energy for work.

To achieve this objective, flow governing equations are formulated, nondimensionalised and approximate solution of the dimensionless coupled nonlinear boundary-value problem are obtained by using a semi-analytical Adomian decomposition method $[22,23]$. The choice of the method is due to the fact that it does not require any linearisation, discretisation, use of initial guess or pertubation. The flow profile, entropy generation rate and irreversibility ratio are then computed.

In the following section, the problem is formulated and non-dimensional analysis is also presented. The entropy generation is presented in Section 3. Section 4 of the work contains the method of solution, results are presented and discussed in Section 5, while Section 6 concludes the paper.

\section{Mathematical Formulation}

The unsteady laminar hydromagnetic non-Newtonian couple stress fluid flow between two permeable and stationary infinite parallel plates is considered as shown in Fig.1. We choose a 2-dimensional cartesian coordinate system with $x$-axis along the flow direction and $y$-axis orthogonal to the planes of the parallel plates, separated by width $y=h$. Fluid injection occurs at the lower plate at a uniform rate $v_{0}$, with a corresponding fluid suction at the upper plate. A constant magnetic field of strength $B_{0}$ is applied perpendicular to the direction of fluid flow. The magnetic Reynolds number is assumed to be small. We further assumed that no external voltage is applied to the flow system, and the induced magnetic field and Hall effect are negligible. In this 


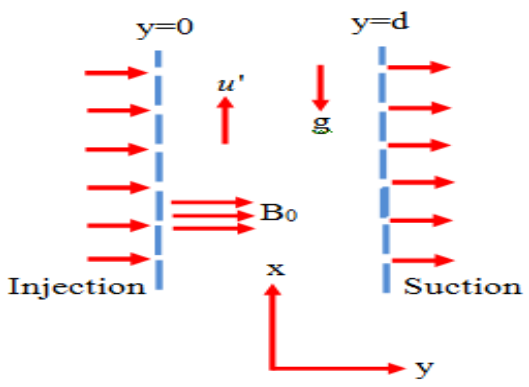

Figure 1: The geometry of the model.

model, we employ the Booussinesq approximation [24], where the gravitational pull on the temperature dependent fluid density acts as a pump for the flow. Using the Stoke's constitutive model for the couple stresses, the momentum balance equation, the energy balance equation, and the local volumetric entropy generation rate $\left(E_{G}\right)$ are given as follows $[25,26]$ :

$$
\begin{gathered}
\rho\left(\frac{d u^{\prime}}{d t^{\prime}}+v_{0} \frac{d u^{\prime}}{d y^{\prime}}\right)=-\frac{d P}{d x^{\prime}}+\mu \frac{d^{2} u^{\prime}}{d y^{\prime 2}}-\eta \frac{d^{4} u^{\prime}}{d y^{\prime} 4}-\sigma_{e} B_{0}^{2} u^{\prime}+\rho g \beta\left(T-T_{0}\right), \\
\rho c_{P}\left(\frac{d T}{d t^{\prime}}+v_{0} \frac{d T}{d y^{\prime}}\right)=\kappa \frac{d^{2} T}{d y^{\prime 2}}+\mu\left(\frac{d u^{\prime}}{d y^{\prime}}\right)^{2}+\eta\left(\frac{d^{2} u^{\prime}}{d y^{\prime 2}}\right)^{2}+\sigma_{e} B_{0}^{2} u^{\prime 2},
\end{gathered}
$$

with the initial boundary conditions

$$
\begin{aligned}
u^{\prime}(0, y) & =v_{0} \sin \left(\frac{\pi}{h} y\right), \\
T(0, y) & =\frac{y^{\prime}}{h}\left(1-\frac{y^{\prime}}{h}\right)+T_{0}, \\
u^{\prime}(0, t) & =u^{\prime}(1, t)=0, \\
T(t, 1) & =T_{0}, \\
\frac{d^{2} u^{\prime}(0, t)}{d y^{\prime 2}} & =\frac{d^{2} u^{\prime}(1, t)}{d y^{\prime 2}}=0,
\end{aligned}
$$

where $u^{\prime}$ and $P$ are the fluid velocity and pressure respectively, $v_{0}$ is the injection/suction velocity at the channel walls, $\eta$ is the coefficient of couple stress, $\mu$ is the dynamic viscosity, $\rho$ is the fluid density, $\sigma_{e}$ is the fluid electrical conductivity, $\kappa$ is the coefficient of thermal conductivity, $c_{P}$ is the isobaric specific heat, $T_{f}$ and $T_{0}$ are referenced fluid temperature, $T$ is the fluid temperature. 
It is often suitable to express the model equations in dimensionless form, which would allow for broad applicability of the equations without having to address the incompatibility that could arise from different unit sys- tems. To this end, we introduce the following dimensionless parameters and variables:

$$
\begin{aligned}
y & =\frac{y^{\prime}}{h}, u=\frac{u^{\prime}}{v_{0}}, t=\frac{v_{0} t^{\prime}}{h}, \theta=\frac{T-T_{0}}{T_{f}-T_{0}}, \\
G & =-\frac{h^{2}}{\mu v_{0}} \frac{\partial P}{\partial x^{\prime}}, R e=\frac{h \rho v_{0}}{\mu}, a^{2}=\frac{\mu h^{2}}{\eta}, \\
P e & =\frac{h \rho C_{p} v_{0}}{\kappa}, H_{a}^{2}=\frac{\sigma_{e} h^{2} B_{0}^{2}}{\mu}, \\
G_{r} & =\frac{g \rho \beta\left(T_{f}-T_{0}\right) h^{2}}{\mu v_{0}}, B r=\frac{v_{0}^{2} \mu}{\kappa\left(T_{f}-T_{0}\right)}, \\
\Omega & =\frac{T_{f}-T_{0}}{T_{0}}, N_{s}=\frac{T_{0}^{2} h^{2} E_{G}}{\kappa\left(T_{f}-T_{0}\right)^{2}},
\end{aligned}
$$

we obtain the following initial boundary value problems

$$
\begin{gathered}
\operatorname{Re}\left(\frac{\partial u}{\partial t}+\frac{\partial u}{\partial y}\right)=G+\frac{\partial^{2} u}{\partial y^{2}}-\frac{1}{a^{2}} \frac{\partial^{4} u}{\partial y^{4}}-H_{a}^{2} u+G_{r} \theta \\
\left(\frac{\partial \theta}{\partial t}+\frac{\partial \theta}{\partial y}\right)=\frac{1}{P e} \frac{\partial^{2} \theta}{\partial y^{2}}+B r\left(\frac{\partial u}{\partial y}\right)^{2}+\frac{B r}{a^{2}}\left(\frac{\partial^{2} u}{\partial y^{2}}\right)^{2}+B r H_{a}^{2} u^{2}
\end{gathered}
$$

with the corresponding dimensionless initial boundary conditions

$$
\begin{aligned}
u(0, y) & =\sin (\pi y), \theta(0, y)=\alpha y(1-y), \\
u(t, 0) & =u(t, 1)=0, \theta(t, 0)=\theta(t, 1)=0, \\
\frac{\partial^{2} u(t, 0)}{\partial y^{2}} & =\frac{\partial^{2} u(t, 1)}{\partial y^{2}}=0,
\end{aligned}
$$

where $\alpha=1 /\left(T_{f}-T_{0}\right)$. In this present work, it is convenient to set $\alpha$ to unity.

\section{Entropy Generation Analysis}

In accordance with the second law of thermodynamics, a common problem in dynamic energy systems such as this is to estimate or forcast the rate of entropy generation as the fluid flows, with the view of eliminating possible degradation 
of the system's useful energy. Irreversibility exists in the flow system due to the fluid motion and heat transfer in the system, which evolves in the interchange of energy and momentum both within the fluid and at the boundaries [27]. For a pseudo 1-D viscous fluid flow in a channel, the inherent flow irreversibility may be analysed using the local volumetric rate of entropy generation, which is given by $[28,29]$ :

$$
E_{G}=\frac{\kappa}{T_{0}^{2}}\left(\frac{d T(t, y)}{d y^{\prime}}\right)^{2}+\frac{\mu}{T_{0}}\left(\frac{d u^{\prime}(t, y)}{d y^{\prime}}\right)^{2}+\frac{\eta}{T_{0}}\left(\frac{\partial^{2} u^{\prime}(t, y)}{\partial y^{2}}\right)^{2}+\frac{\sigma_{e}}{T_{0}} B_{0}^{2} u^{\prime}(t, y)^{2},
$$

where $\sigma_{e}=0$ for a non-conducting fluid. Note that the last term in Eq. (12) is the irreversibility due to heating in the flow system [30]. Using Eqs. (8) and (12), the dimensionless entropy generation function may be expressed as:

$$
N_{s}=\left(\frac{\partial \theta}{\partial y}\right)^{2}+\frac{B_{r}}{\Omega}\left(\frac{\partial u}{\partial y}\right)^{2}+\frac{B_{r}}{\Omega a^{2}}\left(\frac{\partial^{2} u}{\partial y^{2}}\right)^{2}+\frac{B_{r}}{\Omega} H_{a}^{2} u^{2}
$$

The interest here is to obtain the irreversibility ratio to compare the different contributions from the heat energy contributing forces in the fluid system. Notice that the buoyancy force does not contribute any heating effect to the flow. In this respect, we compare the irreversibility due to heat trasfer, $N_{1}$ to the irreversibility due to the combination of the effective viscous effect and the Lorentz force effect, $N_{2}$, where

$$
N_{1}=\left(\frac{\partial \theta}{\partial y}\right)^{2}, N_{2}=\frac{B_{r}}{\Omega}\left(\frac{\partial u}{\partial y}\right)^{2}+\frac{B_{r}}{\Omega a^{2}}\left(\frac{\partial^{2} u}{\partial y^{2}}\right)^{2}+\frac{B_{r}}{\Omega} H_{a}^{2} u^{2},
$$

so that the Bejan number $\left(B_{e}\right)$ is given as

$$
B_{e}=\frac{N_{1}}{N_{s}}=\frac{1}{1+\Phi}
$$

where $\Phi=\frac{N_{2}}{N_{1}}$ is the irreversibility distribution ratio, a parameter that measures the rate of destruction of available work in the flow system. For more detail on the variation $B_{e}$ and the interpretation of ECIS kindly see Ref. [31].

\section{Adomian Decomposition Method of Solution}

To solve the model equations (9) and (10) using the semi-analytical Adomian decomposition scheme, subject to the initial boundary conditions (11), we choose 
the invertible linear differential operator $\frac{\partial}{\partial t}=L_{t}$ such that $L_{t}^{-1}()=.\int_{0}^{t}() d$.$t .$ Following from this, Eqs (9) and (10) may be expressed as

$$
\begin{aligned}
u(t, y) & =F(t, y)+\frac{1}{R_{e}} L_{t}^{-1}\left(Z_{1}-H_{a}^{2} u(t, y)+G_{r} \theta\right) \\
\theta(t, y) & =y(1-y)+L_{t}^{-1} \frac{\partial \theta}{\partial t}
\end{aligned}
$$

where

$$
\begin{aligned}
F(t, y) & =\frac{G t}{R_{e}}+\sin (\pi y) \\
Z_{1} & =-R_{e} \frac{\partial u(t, y)}{\partial y}+\frac{\partial^{2} u(t, y)}{\partial y^{2}}-\frac{1}{R_{e}}\left(\frac{\partial^{2} u(t, y)}{\partial y^{2}}\right)
\end{aligned}
$$

The solutions $u(t, y)$ and $\theta(t, y)$ may be expressed in terms of an infinite series such that

$$
u(y)=\sum_{0}^{\infty} u_{n}(y), \theta(y)=\sum_{0}^{\infty} \theta_{n}(y)
$$

Substituting (20) into (16) and (17), we obtain

$$
\sum_{n=0}^{n=\infty} u_{n}(t, y)=F(t, y)+\frac{1}{R_{e}} L_{t}^{-1}\left(Z_{2} \sum_{n=0}^{n=\infty} u_{n}(t, y)+G_{r} \sum_{n=0}^{n=\infty} \theta_{n}(t, y)\right)
$$

where $Z_{2}=-R_{e} \frac{\partial}{\partial y}+\frac{\partial^{2}}{\partial y^{2}}-\frac{1}{R_{\mathrm{e}}}\left(\frac{\partial^{2}}{\partial y^{2}}\right)-H_{a}^{2}$, and

$$
\sum_{n=0}^{n=\infty} \theta_{n}(t, y)=y(1-y)+L_{t}^{-1}\left(\frac{\partial}{\partial t}\left(\sum_{n=0}^{n=\infty} \theta_{n}(t, y)\right)\right),
$$

from which the recursive Adomian decomposition scheme may be written as

$$
\begin{aligned}
& u_{0}(t, y)=F(t, y) \\
& \theta_{0}(t, y)=y(1-y) \\
& u_{1}(t, y)=\int_{0}^{t}\left(-\frac{\partial u_{0}}{\partial y}+\frac{1}{R_{e}}\left(\frac{\partial^{2} u_{0}}{\partial y^{2}}-\frac{1}{a^{2}} \frac{\partial^{4} u_{0}}{\partial y^{4}}-H_{a}^{2} u_{0}+G_{r} \theta_{0}\right)\right) d t \\
& \theta_{1}(t, y)=\int_{0}^{t}\left(-\frac{\partial \theta_{0}}{\partial y}+\frac{1}{P_{e}} \frac{\partial^{2} \theta_{0}}{\partial y^{2}}+B_{r}\left(\frac{\partial u_{0}}{\partial y}\right)^{2}+\frac{B_{r}}{a^{2}}\left(\frac{\partial^{2} u_{0}}{\partial y^{2}}\right)^{2}+H_{a}^{2} B_{r} u_{0}^{2}\right) d t
\end{aligned}
$$



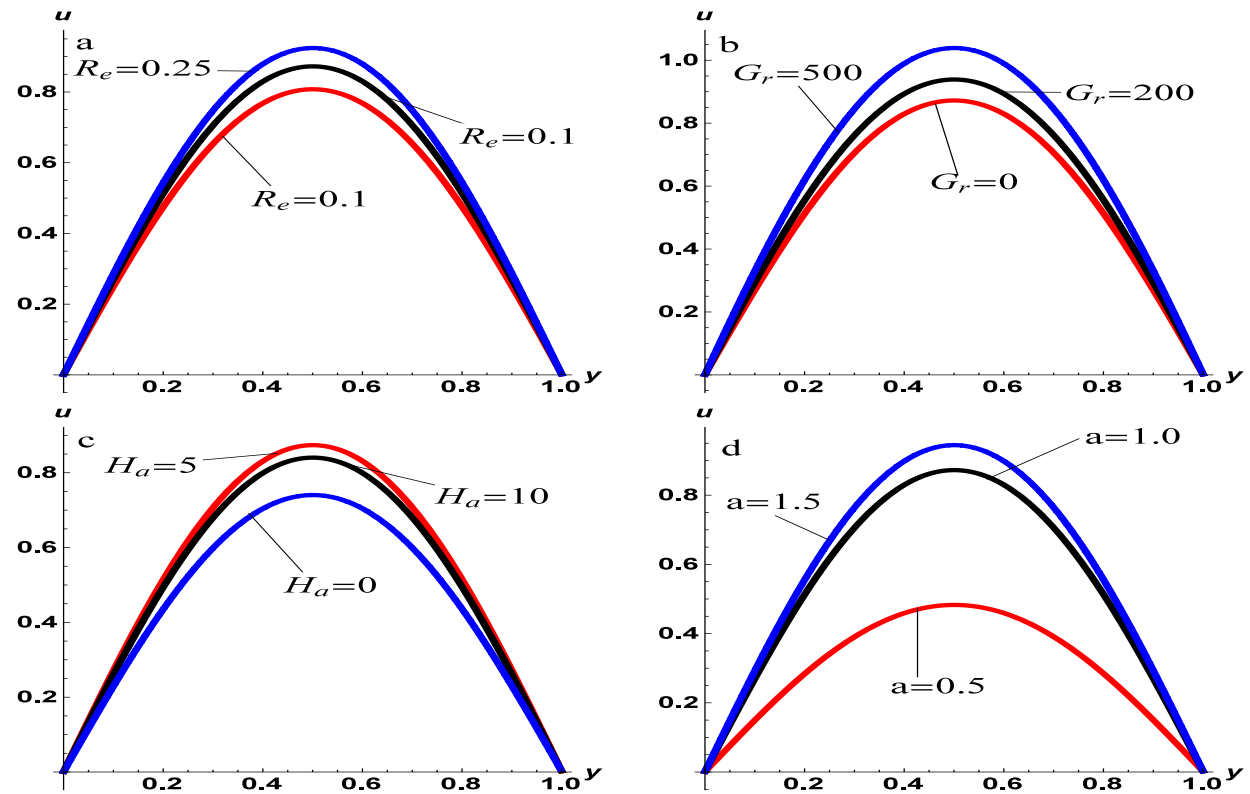

Figure 2: Parameterised velocity profiles of the fluid flow: (a) at varying Reynolds number $R_{e}$, with $P_{e}=0.71, B_{r}=5, G_{r}=1, H_{a}=a=G=1$; (b) at varying Grashoff number $G_{r}$, with $P_{e}=0.71, B_{r}=5, R_{e}=0.15$, $H_{a}=a=G=1$; (c) at varying Hartmann's number $H_{a}$, with $P_{e}=$ $0.71, B_{r}=5, G_{r}=1, a=G=1, R_{e}=0.15$; (d) at varying couple stress inverse $a$, with $P_{e}=0.71, B_{r}=5, G_{r}=1, H_{a}=G=1$

$$
\begin{aligned}
& u_{2}(t, y)=\int_{0}^{t}\left(-\frac{\partial u_{1}}{\partial y}+\frac{1}{R_{e}}\left(\frac{\partial^{2} u_{1}}{\partial y^{2}}-\frac{1}{a^{2}} \frac{\partial^{4} u_{1}}{\partial y^{4}}-H_{a}^{2} u_{1}+G_{r} \theta_{1}\right)\right) d t \\
& \theta_{2}(t, y)=\int_{0}^{t}\left(-\frac{\partial \theta_{1}}{\partial y}+\frac{1}{P_{e}} \frac{\partial^{2} \theta_{1}}{\partial y^{2}}+B_{r}\left(\frac{\partial u_{1}}{\partial y}\right)^{2}+\frac{B_{r}}{a^{2}}\left(\frac{\partial^{2} u_{1}}{\partial y^{2}}\right)^{2}+H_{a}^{2} B_{r} u_{1}^{2}\right) d t \\
& u_{3}(t, y)=\int_{0}^{t}\left(-\frac{\partial u_{2}}{\partial y}+\frac{1}{R_{e}}\left(\frac{\partial^{2} u_{2}}{\partial y^{2}}-\frac{1}{a^{2}} \frac{\partial^{4} u_{2}}{\partial y^{4}}-H_{a}^{2} u_{2}+G_{r} \theta_{2}\right)\right) d t \\
& \theta_{3}(t, y)=\int_{0}^{t}\left(-\frac{\partial \theta_{2}}{\partial y}+\frac{1}{P_{e}} \frac{\partial^{2} \theta_{2}}{\partial y^{2}}+B_{r}\left(\frac{\partial u_{2}}{\partial y}\right)^{2}+\frac{B_{r}}{a^{2}}\left(\frac{\partial^{2} u_{2}}{\partial y^{2}}\right)^{2}+H_{a}^{2} B_{r} u_{2}^{2}\right) d t
\end{aligned}
$$




$$
\begin{aligned}
& u_{k+1}(t, y)=\int_{0}^{t}\left(-\frac{\partial u_{k}}{\partial y}+\frac{1}{R_{e}}\left(\frac{\partial^{2} u_{k}}{\partial y^{2}}-\frac{1}{a^{2}} \frac{\partial^{4} u_{k}}{\partial y^{4}}-H_{a}^{2} u_{k}+G_{r} \theta_{k}\right)\right) d t \\
& \theta_{k+1}(t, y)=\int_{0}^{t}\left(-\frac{\partial \theta_{k}}{\partial y}+\frac{1}{P_{e}} \frac{\partial^{2} \theta_{k}}{\partial y^{2}}+B_{r}\left(\frac{\partial u_{k}}{\partial y}\right)^{2}+\frac{B_{r}}{a^{2}}\left(\frac{\partial^{2} u_{k}}{\partial y^{2}}\right)^{2}+H_{a}^{2} B_{r} u_{k}^{2}\right) d t
\end{aligned}
$$

The Adomian decomposition method has been shown to be a rapidly converging procedure in a number of applications (e.g. Refs. [32, 33]). As a result, only few terms would be required to obtain the approximate solutions of the problem. We set the number of iteration to $m$ so that the approximate solutions (20) may be written as the finite series as follows:

$$
u(t, y)=\sum_{0}^{m} u_{n}(t, y), \theta(t, y)=\sum_{0}^{m} \theta_{n}(t, y)
$$

\section{Results and Discussion}

The dimensionless equations (9)-(13), with the initial and boundary conditions (11) were obtained using the algorithm in Eq. (23) in the limit of Eq. (24), and with codes implemented in MATHEMATICA symbolic package. The velocity, temperature, entropy generation and irreversibility ratio were obtained and displayed to illustrate our findings.

The variations of the dimensionless velocity with the width of the flow channel is presented in Fig.2, for different values of Reynolds parameter $R_{e}$, Grashoff number $G_{r}$, magnetic parameter (Hartmann's number, $H^{2}$ ), and couple stress inverse parameter $a^{2}$. In Fig. $2 \mathrm{a}$, the Reynolds number is directly proportional to the velocity of the fluid body. It is well known that the dynamic viscosity $(\mu)$ in a given flow may diminish with respect to the temperature of the fluid. This could result in inreasing Reynolds number in the fluid motion, which in turn reduces the viscous resistance to the motion. In water for instance, this phenomenon could quickly result in the formation of eddies. In other words, increasing the Reynolds number decreases the effective viscosity in the fluid flow. This effect is similar to the effect of buoyancy on the fluid flow. It is clear from Fig. 2b incresing the Grashof number corresponds to reduction in the viscous forces in the fluid flow. The resultant translational motion of the fluid body 

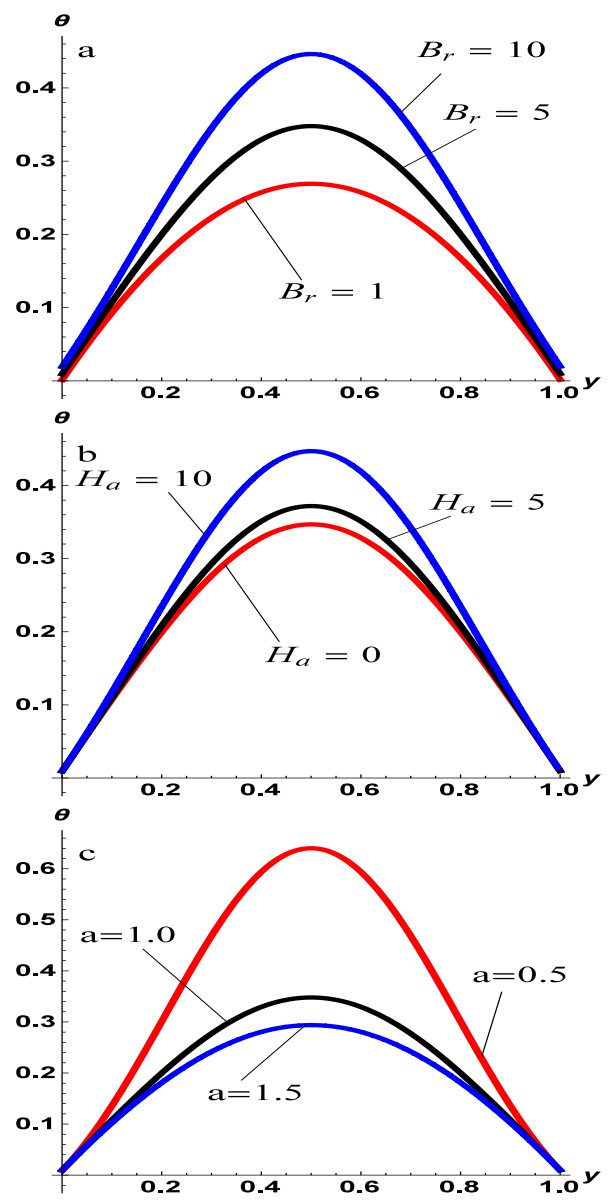

Figure 3: Parametrised temperature profiles of the fluid flow: (a) at varying values of Brinkman number $B r$, with $P_{e}=0.71, R_{e}=0.15$, $G_{r}=1, H_{a}=a=G=1$; (b) at varying values of Hartmann's number $H_{a}$, with $P_{e}=0.71, B_{r}=5, G_{r}=1, a=G=1$; (c) at varying values of couple stress inverse $a$, with $P_{e}=0.71, B_{r}=5, G_{r}=1, H_{a}=G=1$

increases with the Grashof number. In Fig. 2c however, a different phenomenon occurred. Increasing magnetic field, the flow velocity is inhibited. The Lorentz force resulting from the transverse magnetic field constitutes resistance to the momentum of fluid parcels in the adjacent fluid layers, whose presence in the flow system constitutes resistance to the momentum. It is shown in Fig. $2 \mathrm{~d}$ that increasing the couple stress inverse parameter results in increase in the flow velocity. It is observed from the figure that increasing the couple stress inverse 
parameter also increases the resistance to the fluid motion. This friction arises from the effect of particle additve, constituting size dependent effect on couple stress fluids as well as the rotational field of the particles velocity, which is inherent in couple stress fluid. Invariably, an increse in couple stress corresponds to decrease in the velocity profile of the fluid.

In Fig.3, the characteristics of the flow temperature is described with respect to Brinkman number, the magnetic field parameter, and the couple stress parameter in the flow system. It is clear from Fig.3a that increasing the Brinkman number, $B_{r}$ increases the temperature of the fluid. This can be explained by the average decrease in the translational kinetic energy of the flow. The kinetic energy $E=\frac{1}{2} m u^{2}$, in terms of the fluid particle's mass $m$ and velocity $u$. $E$ is comparable with $B_{r}=\frac{1}{2} m v_{0}^{2}$, where $m=\frac{2 \mu}{\kappa\left(T_{\mathrm{f}}-T_{0}\right)}$. In Fig. $3 \mathrm{~b}$, it is observed that increasing the magnetic field intensity results in a decrease in the channel temperature, while Fig.3c shows that increasing couple stresses strenghtens the the intermolecular cohesion in the fluid, which in turn decreases the temperature profile in the channel. This behaviour is expected, because couple stresses cause rotational tendencies in the fluid, which predisposes the flow system to resistance to shear stress.

Furthermore, we examine the effects of the Binkman number $B_{r}$, magnetic parameter, $H^{2}$, couple stress parameter, $a$ on the entropy generation of the flow system, as shown in Fig.4. Fig.4(b) shows that the rate of entropy generation increases with increase Brinkman number, while Fig.4(b) revealed that entropy generation, $N_{s}$ inecreases with increases in the magnetic parameter $H^{2}$. It is well known that viscosity increases as the intermolecular cohesion within the fluid rises. Since the applied Lorentz force inhibits the motion in the fluid, the intermolecular cohesion improves in the fluid resulting in inecrease in entropy generation $N_{s}$ within the channel. Moreover, it is clear from Fig.4(c) that the rate of entropy generation decreases with increase in couple stress parameter.

In Fig.5, we examine the relative dominance of the two forms of irreversibilities that exist in the flow system, which are the irreversibility due to fluid viscosity and ohmic heating, and irreversibility due to heat transfer. We thus found from Fig.5(a-c) that as the parameters $\left(B_{r}\right), H_{a}$ and $a$ increase, the irreversibility due to fluid viscosity dominates the irreversibility distribution ratio.

\section{Conclusion}

Entropy generation rate in unsteady buoyancy-driven hydromagnetic couple stress fluid flow through a porous channel has been investigated. The par- 


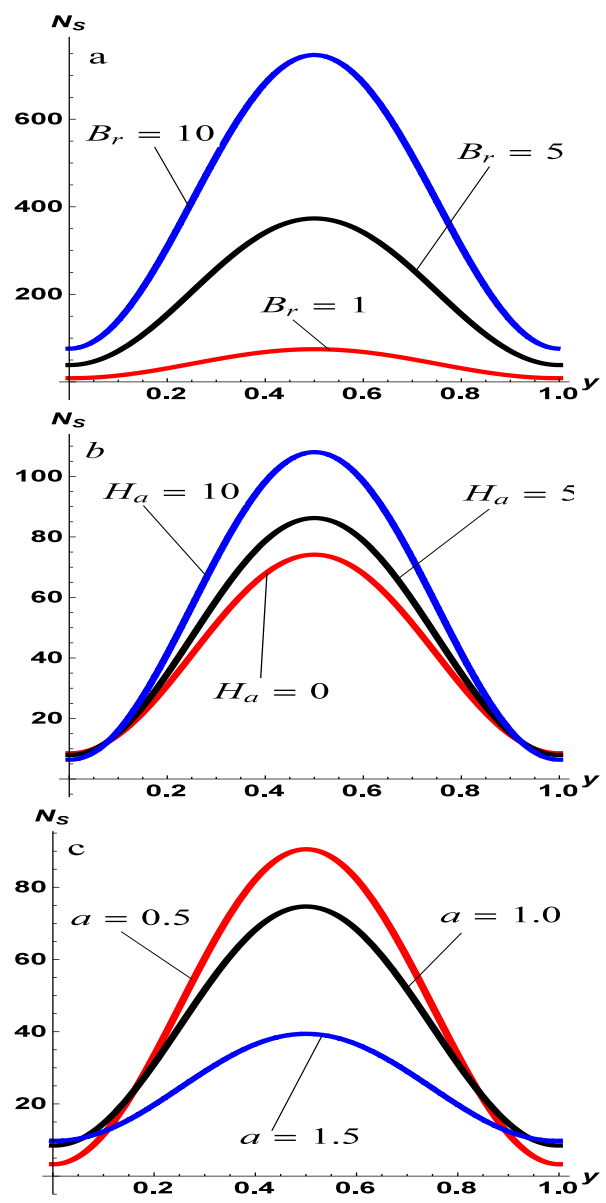

Figure 4: Entropy generation rate: (a) at varying values of Brinkman number $B_{r}$, with $P_{e}=0.71, G_{r}=1, H_{a}=a=G=1$; (b) at varying values of Hartmann's number $H_{a}$, with $P_{e}=0.71, B_{r}=5, G_{r}=1$, $a=G=1$; (c) at varying values of the couple stress inverse $a$, with $P_{e}=0.71, B_{r}=5, G_{r}=1, H_{a} H a=G=1$

tial differential equations are converted into their corresponding dimensionless equivalence, including the prescribed initial boundary conditions. These equations were solved using the Adomian decomposition method and the behaviour of some pertinent fluid variables, such as velocity, temperature, entropy generation rate and the irreversibility ratio were examined and discussed for different parameters of interest, which include, the Grashof number, the Hartmann's 

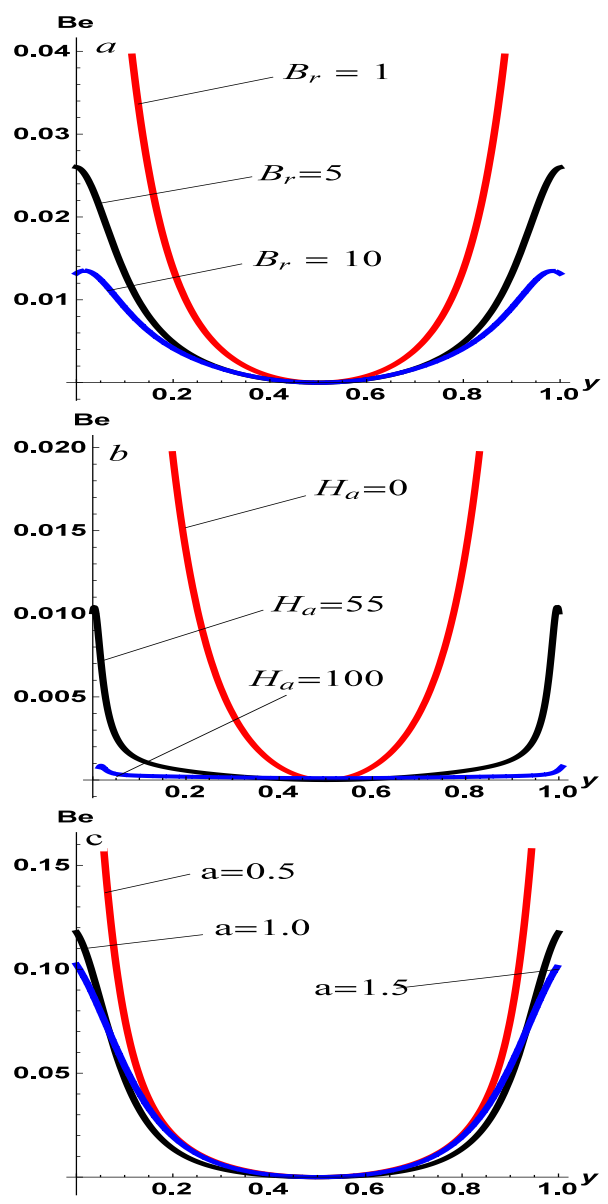

Figure 5: Irreversibility ratio: (a) at varying values of Brinkman number $B_{r}$, with $P_{e}=0.71, G_{r}=1, H_{a}=a=G=1$; (b) at varying values of Hartmann's number $H_{a}$, with $P_{e}=0.71, B_{r}=5, G_{r}=1, a=G=1$; (c) at varying values of the couple stress inverse $a$, with $P_{e}=0.71$, $B_{r}=5, G_{r}=1, H_{a} H a=G=1$

number, the Reynolds number, the Brinkman number and the couple stress parameter.

It was found that the entropy generation rate in the fluid model that has been studied largely depends on the internal forces between the fluid parcels. For example, as the couple stress parameter increases from zero, the electrically conducting fluid builds up more resistance against its flow rate. This has ac- 
counted for the observed increase in the entropy generation rate with respect to the couple stress parameter. Other observed entropy generation trends found their cause to the internal phenomenon in the fluid, though may be triggered by other forces such as the buoyancy and the Lorentz forces. In addition, it was observed that the presence of the Lorentz force in the fluid could be utilise to implement a speed control mechanism in the studied fluid system since increaing magnetic field in the fluid reduces velocity.

\section{Acknowledgements}

UEV is supported by the Royal Society of London, through their Newton International Fellowship Alumni scheme.

\section{References}

[1] M. Devakar, D. Sreennivasu, and B. Shankar. Analytical solutions of couple stress fluid flows with slip boundary conditions. Alexandria Engineering Journal, 53:723-730, 2014.

[2] V.K Stokes. Couple stresses in fluid. Phys. Fluids, 9:1709-1715, 1966.

[3] V.K Stokes. The theory of fluids with microstructure. Spinger, New York, 1984.

[4] S. C. Cowin. The theory of polar fluids. Advances in Applied Mechanics, 14:279-347, 1974.

[5] J. A. Shercliff. The motion of conducting fluids in pipes under transverse magnetic fields. Proceedings of the Cambridge Philosophical Society, 49(1):136-144, 1953.

[6] S. O. Adesanya and O. D. Makinde. Heat transfer to magnetohydrodynamic nonnewtonian couple stress pulsatile flow between two parallel porous plates. Z. Naturforch, a(2012):647-656, 67.

[7] M. Reza and A. S. Gupta. MHD stagnation-point flow of an electrically conducting fluid on the surface of another quiescent fluid. Acta Mechanica, 223(11):23032310, 2012.

[8] S. Das and R. N. Jana. Entopy generation due to MHD flow in a porous channel with navier slip. Ain Shams Eng. J, 5:575-584, 2014.

[9] S. O. Adesanya and J. A. Falade. Thermodynamics analysis of hydromagnetic third grade fluid flow through a channel filled with porous medium. Alexandria Engineering Journal, 54(3):615-622, 2015.

[10] C. K. Chen, B. S. Chen, and C. C. Liu. Entropy generation in mixed convection magnetohydrodynamic nanofluid flow in vertical channel. International Journal of Heat and Mass Transfer, 91:1026 - 1033, 2015.

[11] N. Saeid. Magnetic field effects on entropy generation in heat and mass transfer in porous cavity. Academic J., 8(17):728-739, 2013.

[12] M. Magherbi, A. El-Jery, N. Hidouri, and A. B. Brahim. Evanescent magnetic field effects on entropy generation at the on set of natural convection. Ind. Aca. J., 35(2):163-176, 2010 . 
[13] A. El-Jery, N. Hidouri, M. Magherbi, and A. B. Brahim. Effect of external oriented magnetic field on entropy generation in natural convection. Entropy, 12:1391-1417, 2010.

[14] S. Salas, S. Cuevas, and M. L. Haro. Entropy analysis of magnetohydrodynamic induction devices. J. Phys. D: Appl. Phys., 32:2605-2608, 1999.

[15] S. H. Tasnim and M. A. H. Mahmud. Entropy generation in a porous channel with hydromagnetic effects. Exergy, 2:300-308, 2002.

[16] S. Mahmud, S. H. Tasnim, and H. A. A. Mamun. Thermodynamics analysis of mixed convection in a channel with transverse hydromagnetic effect. Int. J Therm. Sci., 42(731740), 2003.

[17] D. Theuri and O. D. Makinde. Themodynamic analysis of variable viscosity MHD unsteady generalized coutte flow with permeable walls. App. and Comp. Maths, 3(1):1-8, 2014 .

[18] O. Mahian, H. F. Oztop, I. Pop, S. Mahmud, and S. Wongwises. Design of vertical annulus with mhd flow using entropy generation analysis. Therm. Sci., 17(4):1013-1022, 2013.

[19] S. O. Adesanya, S. O. Kareem, J. A. Falade, and S. A. Arekete. Entropy generation analysis for a reactive couple stress fluid flow through a channel saturated with porous material. Energy, 93(1):1239-1245, 2015.

[20] H. A. Attia and Sayed-Ahmed M. E. Hall effect on unsteady MHD couette flow and heat transfer of a bingham fluid with suction and injection. Applied Mathematical Modelling, $28: 10271045,2004$.

[21] S. Das and R. N. Jana. Effect of hall current on entropy generation in porous channel with suction/injection. International Journal of Energy and Technology, 5(25):1-11, 2013.

[22] G. Adomian. Solving Frontier Problems of Physics: The Decomposition Method. Kluwer Academic Publishers, Dordrecht, 1994.

[23] J.S. Duan and R. Rach. A new modification of the adomian decomposition method for solving boundary value problems for higher order nonlinear differential equations. Appl. Math. Comput., 218:4090-4118, 2011.

[24] A. Benjan and A. D. Krauss. Heat transfer handbook. John Wiley, 2003.

[25] O. D. Makinde and T. Chinyoka. Numerical investigation of buoyancy effects on hydromagnetic unsteady flow through a porous channel with suction/injection. Journal of Mechanical Science and Technology, 27(5):1557-1568, 2013.

[26] D. Srinivasacharya and K. Kaladhar. Mixed convection flow of couple stress fluid between parallel vertical plates with hall and ion-slip effects. Commun Nonlinear Sci Numer Simulat, 17:24472462, 2012.

[27] M. H. Mkwizu, O. D. Makinde, and Y. Nkansah-Gyekye. Second law analysis of buoyancy driven unsteady channel flow of nanofluids with convective cooling. Applied and Computational Mathematics, 4(3):100-115, 2015.

[28] L. C. Woods. Thermodynamics of fluid systems. Oxford University Press, Oxford, 1975.

[29] H. F. Oztop and K. Al-Salam. A review on entropy generation in natural and mixed convection heat transfer for energy systems. Renewable and Sustainable Energy Reviews, 16:911-920, 2012. 
[30] S. Das, A. S. Banu, and O. D. Makinde. Entropy analysis on MHD pseudo-plastic nanofluid flow through a vertical porous channel with convective heating. Alexandria Engineering Journal, 54:325337, 2015.

[31] S. O. Kareem, S. O. Adesanya, and U. E. Vincent. Second-law analysis for buoyancydriven hydromagnetic couple stress fluid flow through a porous channel. Comptes Rendus Mcanique, 344(8):547-555, 2016.

[32] S. O. Kareem, S. O. Adesanya, and U. E. Vincent. Second law analysis for hydromagnetic couple stress fluid flow through a porous channel. Alexandria Engineering Journal, 55(2):925-931, 2016.

[33] S. O. Adesanya and O. D. Makinde. Thermodynamic analysis for a third grade fluid through a vertical channel with internal heat generation. J. Hydrodyn., 27(2):264-272, 2015 . 